\title{
Synchronization-Aware Distributed Space-Time Codes in Wireless Relay Networks
}

\author{
Karim G. Seddik, Ahmed K. Sadek, Ahmed S. Ibrahim, and K. J. Ray Liu \\ Department of Electrical and Computer Engineering, \\ and Institute for Systems Research \\ University of Maryland, College Park, MD 20742, USA. \\ \{kseddik, aksadek, asalah, kjrliu\}@eng.umd.edu
}

\begin{abstract}
In this paper, we consider the design of synchronization-aware distributed space-time codes, which we denote as diagonal distributed space-time codes (DDSTC), for $N$ relay nodes helping the source. We impose the diagonal structure of the distributed space-time code to simplify synchronization among the different relay nodes because it is very difficult to synchronize simultaneous transmissions of randomly located relay nodes. We derive an upper bound on the outage probability of the system, which shows that a diversity of order $N$ can be achieved using the diagonal structure of the code. Then, we derive the code design criterion for the DDSTC based on minimizing the pairwise error probability (PEP) to achieve full diversity.
\end{abstract}

\section{INTRODUCTION}

Recently, there has been much interest in modulation techniques to achieve transmit diversity motivated by the increased capacity of multi-input multi-output (MIMO) channels [1]. Severe attenuation in the wireless fading channels makes it crucial to achieve diversity to provide more than one replica of the transmitted symbols to the destination to mitigate the signal degradation due to fading. Because it is difficult to equip the mobile units with more than one antenna with uncorrelated fading, transmit diversity can be achieved through user cooperation leading to what is known as cooperative diversity [2], [3].

Several protocols were proposed in [3] to achieve transmit diversity through user-cooperation such as decode-and-forward and amplify-and-forward protocols. The problem with these protocols is the loss in the data rate as the number of relays increases and this leads to the use of what is known as distributed space-time coding. The term distributed comes from the fact that the virtual multi antenna transmitter is distributed among randomly placed relay nodes. It was proposed in [2] to use relay nodes to form a virtual multi-antenna transmitter to achieve diversity and outage analysis was derived for the system.

Most of the previous works on cooperative transmission assumed perfect synchronization between the nodes, which means that the users' timings, carrier frequencies, and propagation delays are identical. Fig. 1 shows the effect of propagation delay on the received signal from two relays. The sampling time in Fig. 1 is optimal for the first relay signal but clearly it is not optimal for the second relay signal. Some work has been done on selecting the optimal sampling time [4] but this only works for the case of two relays.
In this paper, the design of distributed space-time codes that relax the stringent synchronization requirement is considered. To simplify the synchronization in the network, a diagonal structure is imposed on the space-time code used. The diagonal structure of the code bypasses the perfect synchronization problem by allowing only one relay to transmit at any time slot. Hence, there is no need to synchronize simultaneous "inphase" transmissions of randomly located relay nodes. This greatly simplifies the synchronization since nodes can maintain slot synchronization, which means that coarse slot synchronization is available [5] ${ }^{1}$. However, fine synchronization is more difficult to be achieved. Guard intervals are introduced to ensure that the transmissions from different relays are not overlapped. We allow one relay to consecutively transmit its part of the space-time code from different data packets. This allows us to neglect the overhead introduced by the guard intervals. We derive the code design criterion for the DDSTC. We consider here an amplify-and-forward system model, which simplifies the relay node design and prevents the propagation of relay nodes errors.

Notations. Lower case and upper case bold letters stand for vectors and matrices, respectively. $\operatorname{diag}(\mathbf{a})$, of the $N \times 1$ vector a, is the $N \times N$ diagonal matrix with the elements of the vector a on its diagonal. $\mathbf{I}_{N}$ is the $N \times N$ identity matrix. A $\mathcal{C N}(\mathbf{a}, \mathbf{R})$ random vector is a circularly symmetric complex Gaussian random vector with mean a and covariance matrix R. $\|\mathbf{x}\|_{F}^{2}$ denotes the Frobenius norm of the $L \times 1$ vector $\mathbf{x}$ defined as $\|\mathbf{x}\|_{F}^{2}=\sum_{l=1}^{L}\left|x_{i}\right|^{2}$, where $x_{i}$ is the $i$-th element of the vector $\mathbf{x}$.

\section{System Model}

In this section, we introduce the system model with $N$ relay nodes helping the source by emulating a diagonal space-time code. We consider a two hop system model where there is no direct link from the source to the destination. The channel coefficients from the source to the $n$-th relay is $h_{s, r_{n}}$ and from the $n$-th relay to the destination is $h_{r_{n}, d} . h_{s, r_{n}}$ and $h_{r_{n}, d}$ are modeled as circularly symmetric complex Gaussian random variables with variances $\delta_{s, r_{n}}^{2}$ and $\delta_{r_{n}, d}^{2}$, respectively.

The system has two phases with the time frame structure shown in Fig. 2. In phase 1, the source broadcasts a codeword

\footnotetext{
${ }^{1}$ For example, any synchronization scheme that is used for TDMA systems can be employed to achieve synchronization in the network.
} 


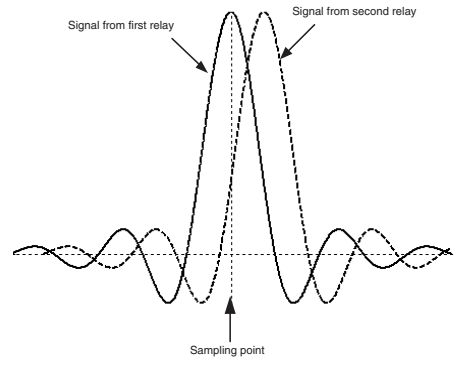

Fig. 1. Baseband signals (each is raised cosine pulse-shaped) from two relays at the receiver.

$\mathbf{x}=\left[x_{1}, x_{2}, \ldots, x_{N}\right]^{T}$ with $E\left\{\|\mathbf{x}\|_{F}^{2}\right\} \leq N$, obtained from a mapping of the source symbols vector $\mathbf{s}=\left[s_{1}, s_{2}, \ldots, s_{N}\right]^{T}$ carved from QAM or PSK constellations, to the $N$ relay nodes. The received signal at the $n$-th relay is

$$
\mathbf{r}_{n}=\sqrt{P_{s}} h_{s, r_{n}} \mathbf{x}+\mathbf{w}_{n}, n=1,2, \cdots, N,
$$

where $P_{s}$ is the transmitted source power and $\mathbf{w}_{n}=$ $\left[w_{n 1}, w_{n 2}, \ldots, w_{n N}\right]^{T}$ denotes the $N \times 1$ additive white Gaussian noise (AWGN) vector at the $n$-th relay and is modeled as $\mathcal{C N}\left(\mathbf{0}, N_{0} \mathbf{I}_{N}\right)$.

In phase 2 , the $n$-th relay multiplies the received signal in phase 1 by the factor

$$
\beta_{n} \leq \sqrt{P_{r} / P_{s}\left|h_{s, r_{n}}\right|^{2}}
$$

to satisfy the power constraint at the relay nodes [3] where $P_{r}$ is the relay node power. The $n$-th relay only forwards the $n$-th element of the received vector (refer to Fig. 2). Hence, the transmitted signal from the $n$-th relay is given by

$$
t_{n}=\beta_{n} \sqrt{P_{x}} h_{s, r_{n}} x_{n}+\beta_{n} w_{n n}, n=1,2, \cdots, N .
$$

The received signal at the destination, due to the $n$-th relay transmission, is given by

$$
\begin{aligned}
y_{n} & =h_{r_{n}, d} \beta_{n} \sqrt{P_{s}} h_{s, r_{n}} x_{n}+h_{r_{n}, d} \beta_{n} w_{n n}+w_{n}^{\prime} \\
& =h_{r_{n}, d} \beta_{n} \sqrt{P_{s}} h_{s, r_{n}} x_{n}+z_{n}, n=1,2, \cdots, N,
\end{aligned}
$$

where $w_{n}^{\prime}$ is modeled as $\mathcal{C N}\left(0, N_{0}\right)$ and hence, $z_{n}$ is $\mathcal{C N}\left(0,\left(\beta_{n}^{2}\left|h_{r_{n}, d}\right|^{2}+1\right) N_{0}\right), n=1,2, \cdots, N$, which accounts for both the noise at the destination node as well as the noise propagating from the relay nodes.

\section{Outage ANALysis FOR the DDSTC BASED RELAY NETWORK}

Define the $N \times 1$ vector $\mathbf{y}=\left[y_{1}, y_{2}, \ldots, y_{N}\right]^{T}$. Due to the diagonal structure of the code, it can be easily verified that the mutual information between the source signal and the received vector satisfies

$$
I_{D D S T C} \leq \sum_{n=1}^{N} \log \left(1+\frac{P_{s} \beta_{n}^{2}\left|h_{r_{n}, d}\right|^{2}\left|h_{s, r_{n}}\right|^{2}}{\left(\beta_{n}^{2}\left|h_{r_{n}, d}\right|^{2}+1\right) N_{0}}\right),
$$
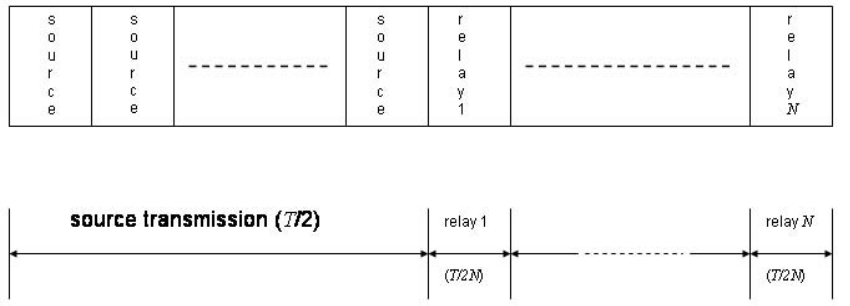

Fig. 2. Time frame structure for the DDSTC based system.

with equality for $x_{n}$ 's independent and zero-mean, circularly symmetric complex Gaussian random variables with unit variance [1].

It is clear that (5) is increasing in $\beta_{i}$ 's, therefore, to maximize the mutual information, the constraint in (2) should be satisfied with equality [3], yielding

$$
I_{D D S T C}=\sum_{n=1}^{N} \log \left(1+f\left(\left|h_{s, r_{n}}\right|^{2} S N R_{1},\left|h_{r_{n}, d}\right|^{2} S N R_{2}\right)\right),
$$

where $S N R_{1}=P_{s} / N_{0}, S N R_{2}=P_{r} / N_{0}$, and

$$
f(v, u)=\frac{u v}{u+v}
$$

Let $P_{s}=P_{r}=P$ and define $S N R=P / N_{0}$, then we can write

$$
I_{D D S T C}=\sum_{n=1}^{N} \log \left(1+f\left(\left|h_{s, r_{n}}\right|^{2} S N R,\left|h_{r_{n}, d}\right|^{2} S N R\right)\right) .
$$

The outage probability for spectral efficiency $R$ (bits/symbol) is defined as

$$
P_{D D S T C}^{\text {out }}(S N R, R)=\operatorname{Pr}\left\{\frac{1}{2} I_{D D S T C}<R\right\},
$$

where the $1 / 2$ factor is due to the loss in the data rate. Equation (7) can be rewritten as

$$
\begin{aligned}
& P_{D D S T C}^{\text {out }}(S N R, R)= \\
& \operatorname{Pr}\left\{\sum_{n=1}^{N} \log \left(1+f\left(\left|h_{s, r_{n}}\right|^{2} S N R,\left|h_{r_{n}, d}\right|^{2} S N R\right)\right)<2 R\right\} .
\end{aligned}
$$

Hence, we have

$$
\begin{aligned}
& P_{D D S T C}^{\text {out }}(S N R, R)= \\
& \qquad \operatorname{Pr}\left\{\sum_{n=1}^{N} \log \left(1+\frac{\left(\left|h_{s, r_{n}}\right|^{2} S N R\right)\left(\left|h_{r_{n}, d}\right|^{2} S N R\right)}{\left|h_{s, r_{n}}\right|^{2} S N R+\left|h_{r_{n}, d}\right|^{2} S N R}\right)<2 R\right\} .
\end{aligned}
$$


To get an upper bound on the outage probability, we have the inequality

$$
\begin{aligned}
\log \left(1+\sum_{n=1}^{N} \frac{\left(\left|h_{s, r_{n}}\right|^{2} S N R\right)\left(\left|h_{r_{n}, d}\right|^{2} S N R\right)}{\left|h_{s, r_{n}}\right|^{2} S N R+\left|h_{r_{n}, d}\right|^{2} S N R}\right) \leq \\
\sum_{n=1}^{N} \log \left(1+\frac{\left(\left|h_{s, r_{n}}\right|^{2} S N R\right)\left(\left|h_{r_{n}, d}\right|^{2} S N R\right)}{\left|h_{s, r_{n}}\right|^{2} S N R+\left|h_{r_{n}, d}\right|^{2} S N R}\right) .
\end{aligned}
$$

Hence, the outage probability can be upper bounded as

$$
\begin{aligned}
& P_{D D S T C}^{\text {out }}(S N R, R) \leq \\
& \operatorname{Pr}\left\{\log \left(1+\sum_{n=1}^{N} \frac{\left(\left|h_{s, r_{n}}\right|^{2} S N R\right)\left(\left|h_{r_{n}, d}\right|^{2} S N R\right)}{\left|h_{s, r_{n}}\right|^{2} S N R+\left|h_{r_{n}, d}\right|^{2} S N R}\right)<2 R\right\} .
\end{aligned}
$$

Define $q_{n}=\frac{\left(\left|h_{s, r_{n}}\right|^{2} S N R\right)\left(\left|h_{r_{n}, d}\right| S N R\right)}{\left|h_{s, r_{n}}\right|^{2} S N R+\left|h_{r_{n}, d}\right| S N R}, n=1, \cdots, N$. The outage probability is now given by

$$
P_{D D S T C}^{\text {out }}(S N R, R) \leq \operatorname{Pr}\left\{\sum_{n=1}^{N} q_{n}<\left(2^{2 R}-1\right)\right\} .
$$

The $q_{n}$ 's for $n=1, \cdots, N$ are the scaled harmonic mean ${ }^{2}$ of two exponential random variables.

The CDF for $q_{n}, n=1, \ldots, N$ is given by [6]

$$
\begin{aligned}
P_{q_{n}}(q) & =\operatorname{Pr}\left\{q_{n}<q\right\} \\
& =1-2 q \sqrt{\zeta_{n 1} \zeta_{n 2}} e^{-q\left(\zeta_{n 1}+\zeta_{n 2}\right)} K_{1}\left(2 q \sqrt{\zeta_{n 1} \zeta_{n 2}}\right),
\end{aligned}
$$

where $\zeta_{n 1}=\frac{1}{S N R \delta_{s, r_{n}}^{2}}, \zeta_{n 2}=\frac{1}{S N R \delta_{r, d}^{2}}$ and $K_{1}($.$) is the$ first order modified Bessel function of the second kind [7]. The function $K_{1}($.$) can be approximated as K_{1}(x) \simeq \frac{1}{x}$ for small $x$ [7] from which we can approximate the CDF of $q_{n}$, at high SNR, as

$$
P_{q_{n}}(q)=\operatorname{Pr}\left\{q_{n}<q\right\} \simeq 1-e^{-q\left(\zeta_{n 1}+\zeta_{n 2}\right)},
$$

which is the CDF of an exponential random variable of rate $\lambda_{n}=\frac{1}{S N R \delta_{s, r_{n}}^{2}}+\frac{1}{S N R \delta_{r_{n}, d}^{2}}$.

Define $Q=\sum_{n=1}^{N} q_{n}$, the CDF of $Q$, assuming the $\lambda_{n}$ 's to be distinct, can be obtained to be

$$
\operatorname{Pr}[Q \leq q]=\sum_{n=1}^{N}\left(\prod_{m=1, m \neq n}^{N} \frac{\lambda_{m}}{\lambda_{m}-\lambda_{n}}\right)\left(1-e^{-\lambda_{n} q}\right) .
$$

The outage probability can be expressed in terms of the CDF of $Q$ as

$$
P_{D D S T C}^{\text {out }}(S N R, R) \leq \operatorname{Pr}\left\{Q<\left(2^{2 R}-1\right)\right\} .
$$

Using the formulation in (17), the outage probability can be proved to satisfy [8]

$$
\begin{aligned}
& P_{D D S T C}^{\text {out }}(S N R, R) \leq \operatorname{Pr}\left[Q<\left(2^{2 R}-1\right)\right] \\
& =\frac{1}{N !}\left(\prod_{n=1}^{N} \lambda_{n}\right)\left(2^{2 R}-1\right)^{N}+\text { H.O.T. }
\end{aligned}
$$

\footnotetext{
${ }^{2}$ The harmonic mean of two numbers, $c_{1}$ and $c_{2}$, equals $\frac{2 c_{1} c_{2}}{c_{1}+c_{2}}$
}

where H.O.T. stands for the higher order terms. Substituting for the $\lambda_{n}$ 's, we get

$$
P_{D D S T C}^{\text {out }}(S N R, R) \lesssim \frac{1}{N !} \cdot \prod_{n=1}^{N} \frac{\delta_{s, r_{n}}^{2}+\delta_{r_{n}, d}^{2}}{\delta_{s, r_{n}}^{2} \delta_{r_{n}, d}^{2}}\left(\frac{2^{2 R}-1}{S N R}\right)^{N}
$$

From the upper bound on the outage probability given by (19), it is clear that the DDSTC based relay network with $N$ relay nodes helping the source will achieve a diversity of order $N$ which is the power of $1 / S N R$.

Also, we can use Jensen's inequality to form an upper bound on the mutual information expression as

$$
\begin{aligned}
I_{D D S T C} & =\sum_{n=1}^{N} \log \left(1+\frac{\left(\left|h_{s, r_{n}}\right|^{2} S N R\right)\left(\left|h_{r_{n}, d}\right|^{2} S N R\right)}{\left|h_{s, r_{n}}\right|^{2} S N R+\left|h_{r_{n}, d}\right|^{2} S N R}\right) \\
& \leq N \log \left(1+\frac{1}{N} \sum_{n=1}^{N} \frac{\left(\left|h_{s, r_{n}}\right|^{2} S N R\right)\left(\left|h_{r_{n}, d}\right|^{2} S N R\right)}{\left|h_{s, r_{n}}\right|^{2} S N R+\left|h_{r_{n}, d}\right|^{2} S N R}\right) .
\end{aligned}
$$

Following a similar approach to the analysis presented in this section, we can easily show that the outage probability of the DDSTC is asymptotically lower bounded by constant $\times$ $S N R^{-N}$. Hence, the outage probability of the DDSTC is asymptotically proportional to $S N R^{-N}$.

\section{DDSTC DESIGN CRITERION}

In this section, we derive the code design criterion of the DDSTC based on the PEP analysis. In the following we set $\beta_{n}=\sqrt{\frac{P_{r}}{P_{s}\left|h_{s, r_{n}}\right|^{2}}}$ to satisfy the power constraint with equality.

Define the variance of $z_{n}$ as

$$
\sigma_{n}^{2}=\left(\frac{P_{r}\left|h_{r_{n}, d}\right|^{2}}{P_{s}\left|h_{s, r_{n}}\right|^{2}}+1\right) N_{0}, n=1, \cdots, N .
$$

The probability density function (pdf) of $\mathbf{y}$ given the $i$-th source symbols vector $\mathbf{s}_{i}$ is given by

$$
\begin{aligned}
& p\left(\mathbf{y} / \mathbf{s}_{i}\right)=\left(\prod_{n=1}^{N} \frac{1}{\pi \sigma_{n}^{2}}\right) \exp \left(-\sum_{n=1}^{N} \frac{1}{\sigma_{n}^{2}}\right. \\
& \left.\left|y_{n}-\sqrt{\frac{P_{s} P_{r}}{P_{s}\left|h_{s, r_{n}}\right|^{2}}} h_{s, r_{n}} h_{r_{n}, d} x_{i n}\right|^{2}\right), i=1, \ldots,|\mathcal{S}|^{N},
\end{aligned}
$$

where $\mathcal{S}$ is the set of possible source symbols, $|\mathcal{S}|$ is the cardinality of $\mathcal{S}$, and $x_{i n}$ is the $n$-th element of the $\mathbf{x}_{i}$ codeword.

The maximum likelihood (ML) decoder can be expressed as

$$
\begin{aligned}
& \arg \max _{\mathbf{s}_{i}} p\left(\mathbf{y} / \mathbf{s}_{i}\right)= \\
& \quad \arg \min _{\mathbf{s}_{i}} \sum_{n=1}^{N} \frac{1}{\sigma_{n}^{2}}\left|y_{n}-\sqrt{\frac{P_{s} P_{r}}{P_{s}\left|h_{s, r_{n}}\right|^{2}}} h_{s, r_{n}} h_{r_{n}, d} x_{i n}\right|^{2} .
\end{aligned}
$$

The PEP of mistaking $\mathbf{s}_{i}$ by $\mathbf{s}_{j}$ is given by [9]

$$
P E P \leq E\left\{\exp \left(\lambda\left[\ln p\left(\mathbf{y} / \mathbf{s}_{j}\right)-\ln p\left(\mathbf{y} / \mathbf{s}_{i}\right)\right]\right)\right\},
$$


and the relation applies for any $\lambda$. From (22), the PEP can be written as

$$
\begin{aligned}
& P E P \leq E_{\mathbf{h}, z_{n}}\left\{\operatorname { e x p } \left(-\lambda\left[\sum_{n=1}^{N} \frac{1}{\sigma_{n}^{2}}\right.\right.\right. \\
& \left(\sqrt{\frac{P_{s} P_{r}}{P_{s}\left|h_{s, r_{n}}\right|^{2}}} h_{s, r_{n}} h_{r_{n}, d}\left(x_{i n}-x_{j n}\right) z_{n}^{*}\right. \\
& +\sqrt{\frac{P_{s} P_{r}}{P_{s}\left|h_{s, r_{n}}\right|^{2}}} h_{s, r_{n}}^{*} h_{r_{n}, d}^{*}\left(x_{i n}-x_{j n}\right)^{*} z_{n} \\
& \left.\left.\left.\left.+\frac{P_{s} P_{r}}{P_{s}\left|h_{s, r_{n}}\right|^{2}}\left|h_{s, r_{n}}\right|^{2}\left|h_{r_{n}, d}\right|^{2}\left|x_{i n}-x_{j n}\right|^{2}\right)\right]\right)\right\}
\end{aligned}
$$

where $\mathbf{h}$ is the channel coefficients vector.

The pdf of $\mathbf{z}$ given the channel coefficients is given by

$$
p_{\mathbf{z} / \mathbf{h}}(\mathbf{z} / \mathbf{h})=\left(\prod_{n=1}^{N} \frac{1}{\pi \sigma_{n}^{2}}\right) \exp \left(-\sum_{n=1}^{N} \frac{1}{\sigma_{n}^{2}} z_{n} z_{n}^{*}\right) \text {. }
$$

Taking the expectations over $\mathbf{z}$ given the channel coefficients we get

$$
\begin{gathered}
P E P \leq E_{\mathbf{h}}\left\{\operatorname { e x p } \left(-\lambda(1-\lambda) \sum_{n=1}^{N} \frac{1}{\sigma_{n}^{2}} \frac{P_{s} P_{r}}{P_{s}\left|h_{s, r_{n}}\right|^{2}}\right.\right. \\
\left.\left.\left(\left|h_{s, r_{n}}\right|^{2}\left|h_{r_{n}, d}\right|^{2}\left|x_{i n}-x_{j n}\right|^{2}\right)\right)\right\} .
\end{gathered}
$$

Choose $\lambda=1 / 2$ that maximizes $\lambda(1-\lambda)$, i.e., minimizing the PEP upper bound. Substituting for $\sigma_{n}^{2}$, the PEP can be upper bounded as

$$
\begin{aligned}
& P E P \leq E_{\mathbf{h}}\left\{\operatorname { e x p } \left(-\frac{1}{4} \sum_{n=1}^{N}\right.\right. \\
& \left.\left.\frac{P_{s}\left|h_{s, r_{n}}\right|^{2} P_{r}\left|h_{r_{n}, d}\right|^{2}}{\left(P_{s}\left|h_{s, r_{n}}\right|^{2}+P_{r}\left|h_{r_{n}, d}\right|^{2}\right) N_{0}}\left(\left|x_{i n}-x_{j n}\right|^{2}\right)\right)\right\} .
\end{aligned}
$$

Define the variable $\gamma_{n}=\frac{P_{s}\left|h_{s, r_{n}}\right|^{2} P_{r}\left|h_{r_{n}, d}\right|^{2}}{\left(P_{s}\left|h_{s, r_{n}}\right|^{2}+P_{r}\left|h_{r_{n}, d}\right|^{2}\right) N_{0}}, \quad n=$ $1, \cdots, N$. Averaging over the channel coefficients, the upper bound on the PEP can be expressed as

$$
P E P \leq \prod_{n=1, x_{i n} \neq x_{j n}}^{N} M_{\gamma_{n}}\left(\frac{1}{4}\left|x_{i n}-x_{j n}\right|^{2}\right),
$$

where $M_{\gamma_{n}}($.$) is the moment generating function (MGF) of$ the random variable $\gamma_{n}$. At high enough signal-to-noise ratio (SNR), the MGF of $\gamma_{n}$ can be approximated as [10]

$$
M_{\gamma_{n}}(s) \simeq \frac{\alpha_{n}}{s}
$$

where

$$
\alpha_{n}=\frac{N_{0}}{P_{s} \delta_{s, r_{n}}^{2}}+\frac{N_{0}}{P_{r} \delta_{r_{n}, d}^{2}} .
$$

Using (30), the PEP can be upper bounded as

$$
\begin{aligned}
& P E P=p\left(\mathbf{s}_{i} \rightarrow \mathbf{s}_{j}\right) \leq N_{0}^{N} . \\
& \prod_{n=1, x_{i n} \neq x_{j n}}^{N}\left(\frac{1}{P_{s} \delta_{s, r_{n}}^{2}}+\frac{1}{P_{r} \delta_{r_{n}, d}^{2}}\right)\left(\frac{1}{4}\left|x_{i n}-x_{j n}\right|^{2}\right)^{-1} .
\end{aligned}
$$

Letting $P_{s}=\alpha P_{t}$ and $P_{r}=(1-\alpha) P_{t}$, where $P_{t}$ is the total power, for some $\alpha \in(0,1)$, we can get the diversity order $d$ of the system as

$$
d=\lim _{P_{t} \rightarrow \infty}-\frac{\log (P E P)}{\log \left(P_{t} / N_{0}\right)}=\operatorname{rank}\left(\Delta\left(\mathbf{x}_{i}, \mathbf{x}_{j}\right)\right),
$$

where $\Delta\left(\mathbf{x}_{i}, \mathbf{x}_{j}\right)=\operatorname{diag}\left(\mathbf{x}_{i}-\mathbf{x}_{j}\right)$.

To achieve a diversity of order $N$, the matrix $\Delta\left(\mathbf{x}_{i}, \mathbf{x}_{j}\right)$ should be of full rank for any $i \neq j$ (that is $x_{i n} \neq x_{j n}, \forall i \neq$ $j, \forall n \in[1, N])$. Furthermore, to minimize the PEP bound in (31) we need to maximize

$$
\min _{\mathbf{x}_{i} \neq \mathbf{x}_{j}}\left(\prod_{n=1}^{N}\left|x_{i n}-x_{j n}\right|^{2}\right)^{1 / N}
$$

which is called the minimum product distance of the set of symbols $\mathbf{s}=\left[s_{1}, s_{2}, \ldots, s_{N}\right]^{T}[11],[12]$.

We will use a linear mapping to form the transmitted codeword, that is

$$
\mathbf{x}=\mathbf{V}_{N \times N} \mathbf{s}
$$

where $\mathbf{s}$ is the source symbols vector. It was proposed in [13], [14], and [15] to use both Hadamard transforms and Vandermonde matrices to design the $\mathbf{V}_{N \times N}$ matrix. The transforms based on the Vandermonde matrices proved to give larger minimum product distance than the Hadamard based transforms. Two classes of optimum transforms were proposed in [13] as follows

1) If $N=2^{k}(k \geq 1)$, the optimum transform is given by

$$
\mathbf{V}_{o p t}=\frac{1}{\sqrt{N}} \operatorname{vander}\left(\theta_{1}, \theta_{2}, \ldots, \theta_{N}\right)
$$

where $\theta_{1}, \theta_{2}, \ldots, \theta_{N}$ are $\theta_{n}=e^{j \frac{4 n-3}{2 N} \pi}, \quad n=$ $1,2, \ldots, N$.

2) If $N=3.2^{k}(k \geq 0)$, the optimum transform is given by

$$
\mathbf{V}_{\text {opt }}=\frac{1}{\sqrt{N}} \operatorname{vander}\left(\theta_{1}, \theta_{2}, \ldots, \theta_{N}\right)
$$

where $\theta_{1}, \theta_{2}, \ldots, \theta_{N}$ are $\theta_{n}=e^{j \frac{6 n-1}{3 N} \pi}, \quad n=$ $1,2, \ldots, N$.

\section{Simulation Results}

In this section, we investigate how the synchronization errors can cause a degradation in the system BER performance. In the simulations, we take the variance of any source-relay or relay-destination channel to be 1 . Fig 3 shows the case of having two relays helping the source and propagation delay mismatch of $T_{2}=0.2 T, 0.4 T$ and $0.6 T$ where $T$ is the time slot duration. We compare the decode-and-forward (DAF) 


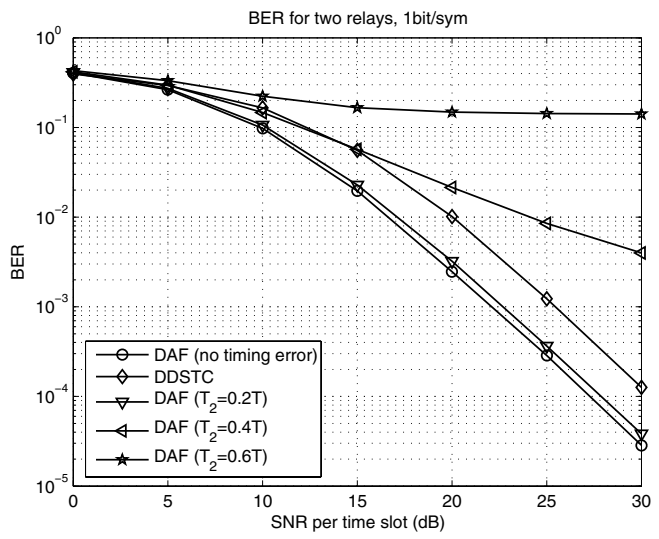

Fig. 3. BER performance with propagation delay mismatch: two relays case.

based on the Alamouti scheme to the DDSTC. We have used raised cosine pulse-shaped waveforms with roll-off factor of 0.2 and QPSK modulation. Clearly, the BER performance of the system highly deteriorates as the propagation delay mismatch becomes larger. Fig 4 shows the case of having three relays helping the source for different propagation delay mismatches. We compare decode-and-forward system (DAF) using the $\mathcal{G}_{3}$ ST block code of [16] and the DDSTC. For fair comparison the number of transmitted bits per symbol is fixed to be $1 \mathrm{bit} / \mathrm{sym}$. Since the $\mathcal{G}_{3}$ ST block code has a data rate of (1/2) [16] which results in an overall system data rate to be (1/3). Therefore, 8-PSK modulation is employed for the system that uses the $\mathcal{G}_{3}$ ST block code. For the DDSTC, QPSK modulation is used as the system has a data rate of $(1 / 2)$. We have used raised cosine pulse-shaped waveforms with roll-off factor of 0.2 . Clearly, the system performance is highly degraded as the propagation delay mismatch becomes larger. From Figures 3 and 4 it is clear that the synchronization errors can highly deteriorate the system BER performance. The DDSTC bypasses this problem by allowing only one relay transmission at any time slot.

\section{CONCLUSION}

In this paper, we consider the design of DDSTC for wireless relay networks. In DDSTC, we impose the diagonal structure of the code to simplify the synchronization among randomly located relay nodes. Synchronization mismatches among the relay nodes cause inter-symbol interference which can highly degrade the system SER performance. DDSTC relaxes the stringent synchronization requirement by allowing only one relay to transmit at any time slot. First, we derive asymptotic upper and lower bounds on the outage probability and prove that the diagonal structure of the code can achieve full diversity of order $N$. Next, we derive, based on PEP analysis, sufficient criterion for the DDSTC to achieve full diversity. The code design criterion turns out to be maximizing the minimum product distance. This is the same criterion used for designing diagonal algebraic space-time (DAST) codes and full-rate fulldiversity space frequency codes.

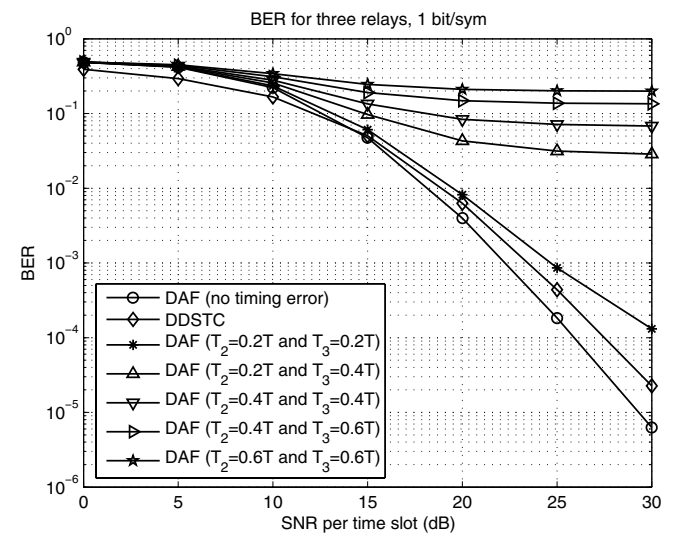

Fig. 4. BER performance with propagation delay mismatch: three relays case.

\section{REFERENCES}

[1] E. Telatar, "Capacity of multi-antenna gaussian channels," European Transactions on Telecommunications, vol. 10, no. 6, pp. 585-595, Nov./Dec. 1999.

[2] J. N. Laneman and G. W. Wornell, "Distributed space-time coded protocols for exploiting cooperative diversity in wireless networks," IEEE Trans. Information Theory, vol. 49, no. 10, pp. 2415-2425, Oct. 2003.

[3] J. N. Laneman, D. N. C. Tse, and G. W. Wornell, "Cooperative diversity in wireless networks: Efficient protocols and outage behavior," IEEE Trans. Information Theory, vol. 50, no. 12, pp. 3062-3080, Dec. 2004.

[4] Y. Mei, Y. Hua, A. Swami, and B. Daneshrad, "Combating synchronization errors in cooperative relays," IEEE International Conference on Acoustics, Speech, and Signal Processing (ICASSP), March 19-23 2005.

[5] X. Li, "Space-time coded multi-transmission among distributed transmitters without perfect synchronization," IEEE Signal Processing Letters, vol. 11, no. 12, pp. 948-951, Dec. 2004.

[6] M. O. Hasna and M. S. Alouini, "End-to-end performance of transmission systems with relays over rayleigh fading channels," IEEE Trans. Wireless Communications, vol. 2, pp. 1126-1131, Nov. 2003.

[7] M. Abramowitz and I. A. Stegun, Handbook of Mathematical Functions with Formulas, Graphs, and Mathematical Tables, Ninth ed., New York, NY: Dover Publications, 1970.

[8] K. Seddik, A. Sadek, W. Su, and K. J. R. Liu, "Outage analysis and optimal power allocation for multi-node relay networks," accepted for publication in IEEE Signal Processing letters.

[9] H. L. Van Trees, Detection, Estimation, and Modulation Theory-Part (I), New York: Wiley, 1968.

[10] W. Su, A. K. Sadek, and K. J. R. Liu, "Cooperative communications in wireless networks: Performance analysis and optimum power allocation," submitted to IEEE Trans. Information Theory, in revision.

[11] C. Schlegel and D. J. Costello Jr., "Bandwidth efficient coding for fading channels: Code construction and performance analysis," IEEE Journal on Selected Areas in Communications, vol. 7, no. 9, pp. 1356-1368, Dec. 1889.

[12] K. Boull and J. C. Belfiore, "Modulation schemes designed for the rayleigh channel," in Proc. CISS92, pp. 288-293, Mar. 1992.

[13] X. Giraud, E. Boutillon, and J. C. Belfiore, "Algebraic tools to build modulation schemes for fading channels," IEEE Trans. Information Theory, vol. 43, no. 3, pp. 938-952, May 1997.

[14] J. Boutros and E. Viterbo, "Signal space diversity: A power- and bandwidth-efficient diversity technique for the rayleigh fading channel," IEEE Trans. Information Theory, vol. 44, no. 4, pp. 1453-1467, July 1998.

[15] Y. Xin, Z. Wang, and G. B. Giannakis, "Space-time diversity systems based on linear constellation precoding," IEEE Trans. on Wireless Communications, vol. 2, no. 2, pp. 294-309, Mar. 2003.

[16] V. Tarokh, H. Jafarkhani, and A.R. Calderbank, "Space-time block coding for wireless communications: Performance results," IEEE Journal on Select Areas in Communications, vol. 17, no. 3, pp. 451-460, Mar. 1999. 